\title{
COSMOsim and COSMOfrag: $a b$ initio computation of bioisosterism and molecular properties with quantum-chemical accuracy at fingerprint speed
} M Thormann*1, A Klamt ${ }^{2}$, K Wichmann ${ }^{2}$, C Wittekindt ${ }^{2}$ and M Almstetter ${ }^{1}$

Address: ${ }^{1}$ Martinsried, Germany and ${ }^{2}$ COSMOlogic GmbH \& Co KG, Burscheider Str. 515, 51381 Leverkusen, Germany

* Corresponding author

from 3rd German Conference on Chemoinformatics

Goslar, Germany. II-13 November 2007

Published: 26 March 2008

Chemistry Central Journal 2008, 2(SuppI I):PI5 doi:10.1186/1752-153X-2-SI-PI5

This abstract is available from: http://www.journal.chemistrycentral.com/content/2/SI/PI5

(c) 2008 Thormann et al.

Theory, validation, and applications of a novel approach for the quantification of drug similarity - COSMOsim are described.

This structure-free method uses the surface polarities as defined in the quantum chemically based conductor-like screening model for realistic solvation (COSMO-RS). The histograms of these surface polarities, the so-called sigma profiles, have been proven sufficient for the accurate calculation of partition and adsorption coefficients and, therefore, of relevant ADMET parameters such as solubility, $\mathrm{pKa}, \log \mathrm{BB}$, and many others.

Sigma profiles carry a large part of the chemophysical information required for the estimation of energetic contributions to desolvation and receptor binding. We demonstrate that two compounds having a high degree of sigma profile similarity, as computed with COSMOsim, have similar physiological action. Therefore, a high COSMOsim value is a necessary condition for bioisosterism. COSMOsim allows seamless scaffold hopping independent from the chemical structure and is, thus, suited for the identification and design of new bioisosteric replacements for functional groups or whole drug candidates.

But aren't quantum chemical calculations too slow for broad virtual screening of millions of compounds? COSMOfrag reduces the computation time by several orders of magnitude while providing sigma profiles of approximate QC quality.
Real life examples will demonstrate the statistical and pharmaceutical plausibility of this approach and its practicability in real drug research projects. 